\title{
ASTEROIDAL DUST AND THE ZODIACAL EMISSION
}

\author{
WILLIAM T. REACH \\ Astronomy Department \\ University of California \\ Berkeley, CA 94720
}

\begin{abstract}
The contribution to the brightness of the infrared background by asteroidal dust, distinguished both by lower color temperature and 'band-pair' morphology, is determined using IRAS observations. Dust band pairs are associated with at least 7 asteroid families and groups, but very little is detected from the remainder of the asteroid belt, indicating that asteroid families and groups are the source of asteroidal dust.
\end{abstract}

\section{Profile-fitting Technique}

The brightness of the $25 \mu \mathrm{m}$ background away from the galactic plane is a smooth function of ecliptic latitude at $0.5^{\circ}$ resolution, with the exception of four bumps at latitudes $\pm 10^{\circ}$ and $\pm 1.4^{\circ}$. These features were first discovered by IRAS (Low et al. 1984), and it was quickly suggested that they are associated with the prominent Hirayama families (Dermott et al. 1984). Using spatial filtering to remove the brightness on angular scales larger than $\sim 3^{\circ}$, Sykes $(1988,1990)$ showed that the inner band pair is itself split into two pairs associated with the Themis and Koronis families; the outer band pair is associated with the Eos family.

We have fit each IRAS profile of the ecliptic with a combination of a smooth (4-th order) polynomial in ecliptic latitude, a linear term in the Galactic H I column density, and 4 Gaussians intended to represent the two main asteroidal band-pairs. Although a pair of Gaussians is not the best model for a band pair (see below), they provide an excellent fit to the data and the center, amplitude, and width allow straightforward interpretation. First, it was immediately evident that the Gaussian widths of the dust bands were well resolved, with full widths at half maximum between 2 and $4^{\circ}$. In contrast, when viewed at higher resolution $\left(2^{\prime}\right)$ in spatially-filtered maps, the inner bands are sharp and should be unresolved at low resolution $\left(30^{\prime}\right)$. Second, the ecliptic latitudes of the four Gaussians used to represent the dust bands reveal a parallactic effect that allows determination of their distance. Due to parallax, the separation between two Gaussians that constitute a band pair is larger for scans made at larger solar elongation. We measured the band-pair separation for a range of solar elongations, and found that the $\pm 10^{\circ}$ (Eos) band pair is 2.4 $\mathrm{AU}$ from the Sun, while the $\pm 1.4^{\circ}$ (Themis \& Koronis blend) band pair is $1.7 \mathrm{AU}$ from the Sun. These distances are clearly smaller than the distances ( 3.0 and $2.9 \mathrm{AU}$, respectively) to the Hirayama families. The sharp dust bands have been shown to be at the same distance as the Hirayama families (Sykes 1990), indicating that the broad emission is produced by dust closer to the Sun than the parent asteroid family.

\section{1}

A.C. Levasseur-Regourd and H. Hasegawa (eds.), Origin and Evolution of Interplanetary Dust, 211-214. C 1991 Kluwer Academic Publishers, Printed in Japan. 


\section{Spectral Decomposition Technique}

In order to determine the total contribution of asteroidal dust to the zodiacal emission (ZE), we have used the IRAS spectrum at each position in the sky to separate the infrared background into 3 components: 1) relatively hot dust near the Earth's orbit that dominates the $\mathrm{ZE}, 2$ ) warm dust that is in the asteroid belt, and 3) cold dust in the interstellar medium. Three spectral components allow one extra degree of freedom in fitting the four IRAS wavebands at $12,25,60$, and $100 \mu \mathrm{m}$. The spectrum of the interplanetary components was calculated using the size distribution of particles near the Earth's orbit (Grün et al. 1985) and the optical properties of silicates (see Reach 1988). It was necessary to correct the color of the 'hot' component for lines of sight with different solar elongation and latitude; the corrections were most important at $12 \mu \mathrm{m}$ (in the Wien portion of the spectrum).

Maps of the three separate components of the infrared background clearly show the different morphology of the respective emitting regions: the hot component is very smooth, the warm component shows the parallel zodiacal dust bands, and the cold component shows the interstellar cirrus. A slice through the asteroidal component from the North to the South ecliptic poles at $90^{\circ}$ solar elongation is shown in Figure 1. Although the brightness of the asteroidal component is less than $3 \%$ of the $\mathrm{ZE}$ brightness, the point-to-point noise is only about $0.1 \%$ of the $\mathrm{ZE}$ brightness. In Figure 1 , the twin bumps at $\pm 10^{\circ}$, representing the Eos band pair, and the central, marginally resolved bumps at $\pm 1.4^{\circ}$, representing the blended Themis \& Koronis band pairs, are clearly evident.

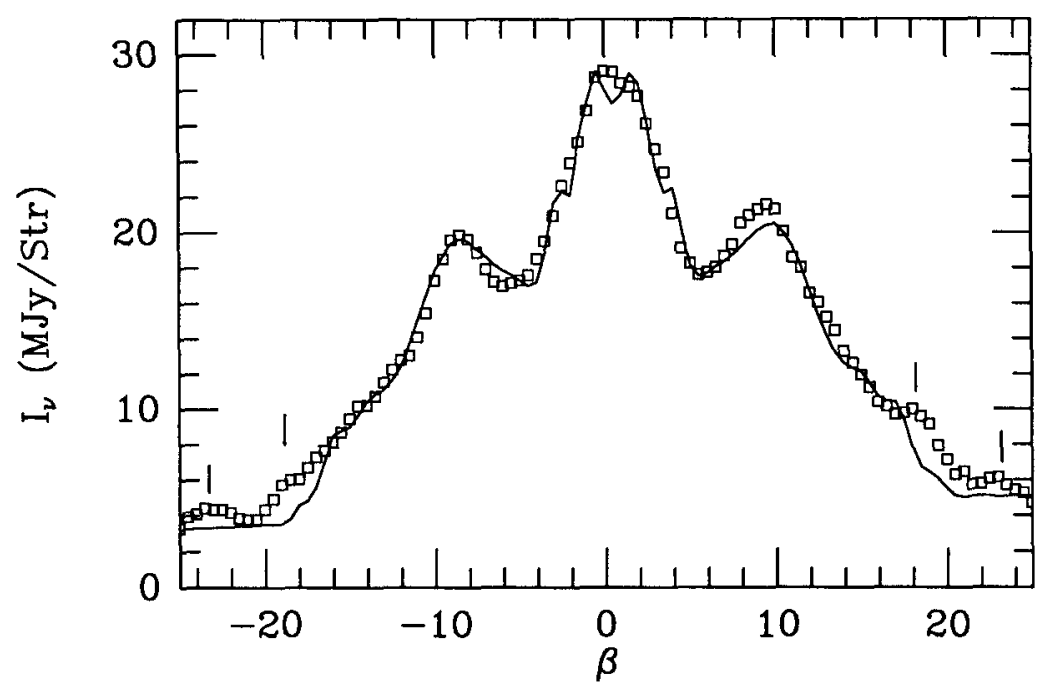

Figure 1. The $25 \mu \mathrm{m}$ surface brightness of the asteroidal component of the zodiacal emission as a function of ecliptic latitude, for solar elongation $90^{\circ}$. The observations (squares) are shown together with the asteroid family fit (curve). Two band pairs not associated with prominent families are indicated by vertical lines; the $\pm 24^{\circ}$ pair is probably associated with the Phocaea group. 
Most interesting in the map of the asteroidal component of the $\mathrm{ZE}$ is the presence of apparent broad band pairs at a range of ecliptic latitudes. The band pairs discovered by Sykes (1988) in spatially filtered maps are confirmed, as well as new bands. Since the bands are severely blended, it is not possible to uniquely disentangle the emission of the separate bands. We have calculated the theoretical brightness profile (Sykes 1990) of band pairs associated with the main asteroid families, and used a least-squares fit to determine the amount of emission contributed by each family. The only free parameter in the fits was the total surface area of grains associated with each family. The theoretical band pair was convolved to $0.5^{\circ}$ resolution, and the dispersion in the orbital elements was boosted for each family by factors of 1 (Themis), 2 (Eos), and 3 (all others) to match the observed band-pair separations. In addition to the asteroid family models, a Gaussian with variable amplitude, center, and width was included as a model for the non-family, main belt asteroidal dust. The Gaussian was unable to fit the observations better than the dust band models, despite a wide range of guesses for its initial conditions; we place an upper limit of $0.6 \mathrm{MJy} / \mathrm{Str}$ on the brightness of the dust associated with non-family asteroids. The least-squares fit is shown as a smooth solid line in Figure 1, and the surface areas are given in Table 1.

TABLE 1. Asteroidal dust band surface areas

\begin{tabular}{lc}
\hline Family & Surface Area $\left(10^{19} \mathrm{~cm}^{2}\right)$ \\
\hline Themis/Koronis/Nysa & $2.0 \pm .3$ \\
Flora & $0.9 \pm .3$ \\
Eos & $58 . \pm 1$. \\
Io & $1.9 \pm .4$ \\
Maria & $1.2 \pm .3$ \\
Phocaea & $1.7 \pm .5$ \\
\hline
\end{tabular}

The results in Table 1 may not represent a unique solution for the amount of emission from each asteroid family, due to blending of some of the bands. The Themis/Koronis/Nysa blend is very severe. We find that the amount of dust in the Themis and Nysa bands is greater than in the narrower Koronis band, but it is difficult to determine how much of the Themis band is actually produced by a broad component of the Koronis band. Sykes (1990) has shown that the Koronis band is much brighter than the Themis band, when viewed at high angular resolution. Some new features are evident in the residuals from the fits. We can tentatively associate the bumps at $\pm 21^{\circ}$ (visible at the edges of Figure 1 ) with the Phocaea group of asteroids, which is not considered a true family but a grouping bounded by orbital instabilities (see Valsecchi et al. 1989). If this association is real, then the dust bands are not necessarily relics of the collisions that produced true asteroid families.

\section{Discussion}

The predominance of asteroid families and groups as regions of active dust production indicates that the collisional environment in the asteroid belt is not uniform: family asteroids constitute some $26 \%$ of the numbered asteroids, but the actual fraction of all asteroids is 
likely to be closer to $4 \%$ when selection effects in asteroid searches are taken into account (Valsecchi et al. 1989). The $20-200 \mu \mathrm{m}$ size grains that emit the infrared radiation detected by IRAS are comminution results of relatively recent collisions, less than $10^{6}$ yrs ago based on Poynting-Robertson lifetimes. If asteroids are indeed the source of a large fraction of the interplanetary dust that is near the Earth's orbit, it would be expected that dynamical signatures of family origin will be discovered; in the case of true family origin (as opposed to origin in the Phocaea group), chemical signatures may also be discovered.

In the ecliptic plane, the total asteroidal dust area per unit volume $\langle n \sigma\rangle=2.7 \times 10^{-22}$ $\mathrm{cm}^{2} / \mathrm{cm}^{3}$. For comparison, near the Earth's orbit $\langle n \sigma\rangle$ is about 1.7 times (Grün et al. 1985) to 3 times (Reach 1988) larger. In steady state, Poynting-Robertson (PR) drag spreads the dust through the inner Solar System with the volume density increasing as inversely as the heliocentric distance- a factor of 3 decrease between the Earth's orbit and the asteroid belt. Thus the asteroidal dust bands have at least enough dust to maintain the interplanetary dust complex. The loss rate from a given family will decrease with time, as the larger, less-abundant particles (with longer PR time scales) drift inward. It is presently uncertain whether new particles are continuously being generated in asteroid families to balance the losses due to PR drag.

\section{References}

Dermott, S.F., Nicholson, P.D., Burns, J.A., and Houck, J.R. (1984) 'Origin of the Solar System dust bands discovered by IRAS', Nature 312, 505 .

Grün, E., Zook, H. A., Fechtig, H., and Giese, R.H. (1985) 'Collisional balance of the meteoritic complex', Icarus 62, 244.

Hauser, M.G. (1988) 'Models for infrared emission from zodiacal dust', in A. Lawrence (ed.), Comets to Cosmology, Springer-Verlag, Berlin, pp. 27-39.

Low, F.J. et al. (1984) 'Infrared cirrus: New components of the extended infrared emission', $A p$. J. 278, L19.

Reach, W.T. (1988) 'Zodiacal emission I. Dust near the Earth's orbit', Ap. J. 335, 468.

Reach, W.T. (1991) 'Zodiacal emission II. Dust near the Ecliptic', Ap. J. 387, in press.

Reach, W.T. and Heiles, C. (1988) 'Separating the Solar System and Galactic contributions to the diffuse infrared background', in A. Lawrence (ed.), Comets to Cosmology, Springer-Verlag, Berlin, pp. 40-43.

Sykes, M.V. (1988) 'IRAS observations of extended zodiacal structures', Ap. J. 334, L55.

Sykes, M.V. (1990) 'Zodiacal dust bands: Their relation to asteroid families', Icarus 84, 267.

Valsecchi, G.B., Carusi, A., Knezevic, Z., Kresak, L., and Williams, J.G. (1989) 'Identification of asteroid families', in R.P. Binzel, T. Gehrels, and M.S. Matthews (eds.), Asteroids II, University of Arizona Press, Tucson, pp. 368-385. 